\title{
Synthesis of some novel hydrazone derivatives and evaluation of their antituberculosis activity
}

\author{
Ahmet Özdemir1, Zafer Asım Kaplancıklı1, Gülhan Turan-Zitouni1, Gilbert Revial2
}

\begin{abstract}
The heterocyclic hydrazone constitute an important class of biologically active drug molecules which have attractive attention of medicinal chemists due to their antituberculosis activities. For this purpose, new hydrazone derivatives were synthesized and evaluated for antituberculosis activity. The reaction of $(5,6,7,8$-tetrahydronaphthalen-1-yl) acetic acid hydrazide with various benzaldehydes gave 5,6,7,8-tetrahydronaphtalen acetic acid benzylidene hydrazide derivatives. The chemical structures of the compounds were elucidated by $1 \mathrm{H}-\mathrm{NMR}$, El-MS spectral data and Elemental Analysis. The compounds were evaluated for antituberculosis activity against Mycobacterium tuberculosis H37Rv (ATCC 27294) using the BACTEC 460 radiometric system and BACTEC 12B medium. The preliminary results indicated that all of the tested compounds showed low activity against the test organism. The compound A10 showed high antituberculosis activity (IC50: $3.072 \mu \mathrm{g} / \mathrm{mL}$ and IC90: $3.358 \mu \mathrm{g} / \mathrm{mL}$ ) and low cytotoxicity (CC50: $>40 \mu \mathrm{g} / \mathrm{mL}$ ).
\end{abstract}

KEY WORDS: Hydrazone, Antituberculosis activity, Mycobacterium tuberculosis

\section{INTRODUCTION}

In spite of a 5000 year history, tuberculosis (TB) remains the leading single-agent infectious disease killer in the world. Approximately one third of the world's population is infected with TB bacilli, and each year almost 8 million people develop active TB and 2 million die as a result of TB. The major challenges for tuberculosis control are the development of multidrug-resistant tuberculosis (MDRTB) strains and the increasing numbers of immunocompromised

individuals with HIV infections who are highly susceptible to the disease. As a result, there is a pressing need for new antitubercular agents acting with greater potency and efficacy than the current existing drugs (1).

To pursue this goal, our research efforts are directed to find new chemical classes of antimycobacterially active agents. The methods of investigation of structure-activity relationships (SARs) enabled us to find some new pharmacophores of the above-mentioned activity. Many studies were carried out on heterocyclic systems bearing a hydrazone structure as a pharmacophore (2-13). In this study, we planned to synthesize new mole- cules bearing hydrazone moieties for their potential antituberculosis activity.

\section{Chemistry}

The synthetic route of the compounds is outlined in Scheme 1. For the synthesis of the title compounds, 5,6,7,8-tetrahydronaphthalene acetic acid hydrazide required as starting material was prepared by the reaction of 5,6,7,8-tetrahydronaphthalene acetic acid ethyl ester with hydrazine hydrate (14). The reaction of equimolar quantities of hydrazide with appropriate benzaldehydes in the presence of isopropyl alcohol resulted in the formation of the title compounds (A1-15) (Table 1).

\section{Pharmacology}

Antituberculosis activity and Cytotoxicity

The initial screen is conducted against Mycobacterium tuberculosis H37Rv (ATCC 27294) in BACTEC 12B medium using the Microplate Alamar Blue Assay (MABA) (15). One of the compounds showed significant antituberculosis activity as can be inferred from Table 2 .

The VERO cell cytotoxicity assay (16) is done in parallel with the TB Dose Response assay. Viabil-
AFFILIATIONS

${ }^{1}$ Anadolu Üniversitesi

Eczacılık Fakültesi,

Farmasötik Kimya, Eskişehir

Türkiye

2UMR-CNRS 7084, Cnam, 2

rue Conte', 75003 , Laboratoire

de Transformations Chimiques

et Pharmaceutiques, Paris,

France

CORRESPONDENCE

Ahmet Özdemir

E-mail:

ahmeto@anadolu.edu.tr

Received:

February 01, 2010

Accepted:

March 07, 2010

*Author to whom

correspondence should

be addressed; e-mail:

ahmet0@anadolu.edu.tr, phone: +90222 3350580

/ 3774, fax: +90222 335

0750. 


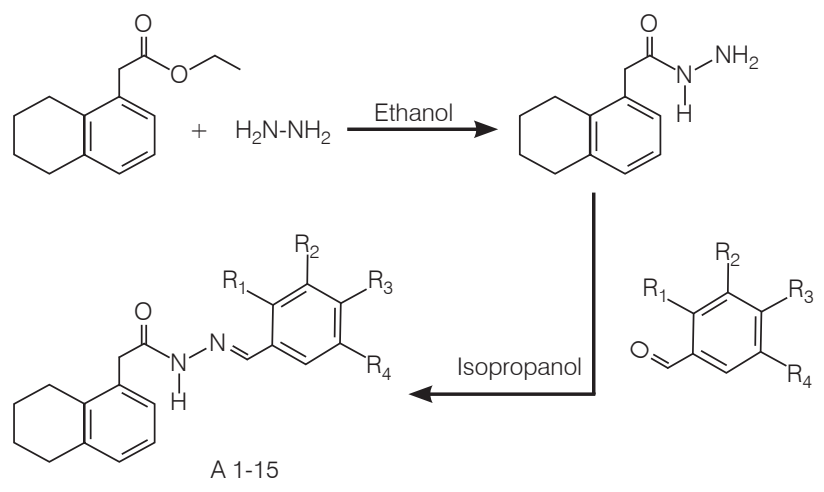

SCHEME 1. Synthetic protocol of the title compounds

ity is assessed using Promega's Cell Titer-Glo Luminescent Cell Viability Assay (17).

\section{EXPERIMENTAL}

\section{Chemistry}

All melting points (m.p.) were determined in open capillaries on a Gallenkamp apparatus and are uncorrected. The purity of the compounds was routinely checked by thin layer chromatography (TLC) using silica gel 60G (Merck). Spectroscopic data were recorded on the following instruments: ${ }^{1} \mathrm{H}$ NMR, Bruker $400 \mathrm{MHz}$ NMR spectrometer in DMSO- $\mathrm{d}_{6}$ using TMS as an internal standard; elemental analyses were performed on a Perkin Elmer EAL 240 elemental analyser; EI-MS, VG Quattro mass spectrometer.

\section{Preparation of 5,6,7,8-tetrahydronaphthalene acetic acid hydrazide}

In a flask equipped with a reflux condenser, a mixture of 5,6,7,8-tetrahydronaphthalene acetic acid ethyl ester (100 $\mathrm{mmol})$ and the hydrazine hydrate $(100 \mathrm{mmol})$ is reacted in ethanol $(200 \mathrm{~mL})$. The mixture is then refluxed for $1 \mathrm{~h}$ and the obtained solid is filtered and used without further purification (14).

\section{Preparation of 5,6,7,8-tetrahydronaphtalen acetic acid benzylidene hydrazide $\mathrm{A} 1-15$}

The reaction of equimolar quantities of hydrazide $(5 \mathrm{mmol})$ with appropriate benzaldehyde $(5 \mathrm{mmol})$ in the presence of isopropyl alcohol resulted in the formation of the title compounds. Some characteristics of the synthesized compounds are shown in Table 1.

A1: ${ }^{1} \mathrm{H}-\mathrm{NMR}: \delta$ 1.60-1.80 $(4 \mathrm{H}, \mathrm{m}), 2.60-2.75(4 \mathrm{H}, \mathrm{m}), 3.74(2 \mathrm{H}$, s), $6.90-7.10(3 \mathrm{H}, \mathrm{m}), 7.49(2 \mathrm{H}, \mathrm{d}, J=8.5 \mathrm{~Hz}), 7.70(2 \mathrm{H}, \mathrm{dd}, J=8.5$, $2.1 \mathrm{~Hz}), 8.12(1 \mathrm{H}, \mathrm{s}), 11.51(1 \mathrm{H}, \mathrm{s})$. EIMS $(\mathrm{m} / \mathrm{z}): 326\left(\mathrm{M}^{+}, 9.5 \%\right)$, 291 (1), 188 (27), 181 (8), 171 (25), 154 (17), 145 (100), 140 (58), 129 (31). For $\mathrm{C}_{19} \mathrm{H}_{19} \mathrm{ClN}_{2} \mathrm{O}$ calculated: $69.83 \%$ C, $5.86 \%$ H, 8.57 \% N; found: $69.85 \%$ C, $5.89 \% \mathrm{H}, 8.56 \% \mathrm{~N}$.

A2: ${ }^{1} \mathrm{H}-\mathrm{NMR}: \delta$ 1.65-1.80 $(4 \mathrm{H}, \mathrm{m}), 2.33(3 \mathrm{H}, \mathrm{s}), 2.65-2.75(4 \mathrm{H}$, $\mathrm{m}), 3.71(2 \mathrm{H}, \mathrm{s}), 6.90-7.10(3 \mathrm{H}, \mathrm{m}), 7.24(2 \mathrm{H}, \mathrm{d}, J=8.0 \mathrm{~Hz}), 7.57$ $(2 \mathrm{H}, \mathrm{dd}, J=8.0,2.0 \mathrm{~Hz}), 8.08(1 \mathrm{H}, \mathrm{s}), 11.37(1 \mathrm{H}, \mathrm{s})$. EIMS $(\mathrm{m} / \mathrm{z})$ : $306\left(\mathrm{M}^{+}, 31 \%\right), 288$ (9), 262 (1), 248 (1), 188 (30), 171 (25), 161 (31), 145 (100), 134 (37), 120 (40). For $\mathrm{C}_{20} \mathrm{H}_{22} \mathrm{~N}_{2} \mathrm{O}$ calculated: $78.40 \%$ C, $7.24 \%$ H, 9.14 \% N; found: $78.44 \%$ C, $7.25 \%$ H, 9.15 $\% \mathrm{~N}$.

A3: ${ }^{1} \mathrm{H}-\mathrm{NMR}: \delta$ 1.65-1.80 $(4 \mathrm{H}, \mathrm{m}), 2.65-2.75(4 \mathrm{H}, \mathrm{m}), 3.72(2 \mathrm{H}$, s), $3.80(3 \mathrm{H}, \mathrm{s}), 6.90-7.05(5 \mathrm{H}, \mathrm{m}), 7.62(2 \mathrm{H}, \mathrm{d}, \mathrm{J}=6.0 \mathrm{~Hz}), 8.06$ $(1 \mathrm{H}, \mathrm{s}), 11.30(1 \mathrm{H}, \mathrm{s})$. EIMS $(\mathrm{m} / \mathrm{z}): 322\left(\mathrm{M}^{+}, 38\right.$ \%), $188(21), 177$ (22), 171 (15), 150 (56), 145 (100), 135 (26), 131 (26). For $\mathrm{C}_{20} \mathrm{H}-$ ${ }_{22} \mathrm{~N}_{2} \mathrm{O}_{2}$ calculated: $74.51 \% \mathrm{C}, 6.88 \% \mathrm{H}, 8.69 \% \mathrm{~N}$; found: 74.52 $\%$ C, $6.90 \% \mathrm{H}, 8.71 \% \mathrm{~N}$.

A4: ${ }^{1} \mathrm{H}-\mathrm{NMR}: \delta$ 1.65-1.80 (4H, m), 2.60-2.75 $(4 \mathrm{H}, \mathrm{m}), 3.79(2 \mathrm{H}$, s), 6.90-7.07 (3H, m), $7.94(2 \mathrm{H}, \mathrm{d}, J=8.8 \mathrm{~Hz}), 8.27(2 \mathrm{H}, \mathrm{d}, J=8.8$ $\mathrm{Hz}), 8.23(1 \mathrm{H}, \mathrm{s}), 11.73(1 \mathrm{H}, \mathrm{s})$. EIMS $(\mathrm{m} / \mathrm{z}): 337\left(\mathrm{M}^{+}, 2\right.$ \%), 320

TABLE 1. Some characteristics of the compound

\begin{tabular}{|c|c|c|c|c|c|c|c|c|}
\hline Comp. & $\mathbf{R}_{1}$ & $\mathbf{R}_{2}$ & $\mathbf{R}_{3}$ & $\mathbf{R}_{4}$ & YIELD \% & M.P. ${ }^{\circ} \mathbf{C}$ & MOL. FORMULA & M.W. \\
\hline A1 & $\mathrm{H}$ & $\mathrm{H}$ & $\mathrm{Cl}$ & $\mathrm{H}$ & 78 & $192-194$ & $\mathrm{C}_{19} \mathrm{H}_{19} \mathrm{CIN}_{2} \mathrm{O}$ & 326 \\
\hline $\mathrm{A} 2$ & $\mathrm{H}$ & $\mathrm{H}$ & $\mathrm{CH}_{3}$ & $\mathrm{H}$ & 80 & $164-165$ & $\mathrm{C}_{20} \mathrm{H}_{22} \mathrm{~N}_{2} \mathrm{O}$ & 306 \\
\hline A3 & $\mathrm{H}$ & $\mathrm{H}$ & $\mathrm{OCH}_{3}$ & $\mathrm{H}$ & 82 & $154-156$ & $\mathrm{C}_{20} \mathrm{H}_{22} \mathrm{~N}_{2} \mathrm{O}_{2}$ & 322 \\
\hline A4 & $\mathrm{H}$ & $\mathrm{H}$ & $\mathrm{NO}_{2}$ & $\mathrm{H}$ & 79 & 205-206 & $\mathrm{C}_{19} \mathrm{H}_{19} \mathrm{~N}_{3} \mathrm{O}_{3}$ & 337 \\
\hline A5 & $\mathrm{H}$ & $\mathrm{H}$ & $\mathrm{CN}$ & $\mathrm{H}$ & 75 & $211-212$ & $\mathrm{C}_{20} \mathrm{H}_{19} \mathrm{~N}_{3} \mathrm{O}$ & 317 \\
\hline A6 & $\mathrm{H}$ & $\mathrm{H}$ & $\mathrm{CH}\left(\mathrm{CH}_{3}\right)_{2}$ & $\mathrm{H}$ & 80 & $151-152$ & $\mathrm{C}_{22} \mathrm{H}_{26} \mathrm{~N}_{2} \mathrm{O}$ & 334 \\
\hline A7 & $\mathrm{H}$ & $\mathrm{H}$ & $\mathrm{OCH}_{2} \mathrm{C}_{6} \mathrm{H}_{5}$ & $\mathrm{H}$ & 85 & $165-166$ & $\mathrm{C}_{26} \mathrm{H}_{26} \mathrm{~N}_{2} \mathrm{O}_{2}$ & 398 \\
\hline A8 & $\mathrm{H}$ & $\mathrm{H}$ & $\mathrm{Cl}$ & $\mathrm{Cl}$ & 88 & $196-197$ & $\mathrm{C}_{19} \mathrm{H}_{18} \mathrm{Cl}_{2} \mathrm{~N}_{2} \mathrm{O}$ & 360 \\
\hline A9 & $\mathrm{H}$ & $\mathrm{H}$ & $\mathrm{OH}$ & $\mathrm{OCH}_{3}$ & 65 & 191-192 & $\mathrm{C}_{20} \mathrm{H}_{22} \mathrm{~N}_{2} \mathrm{O}_{3}$ & 338 \\
\hline A10 & $\mathrm{H}$ & $\mathrm{H}$ & $\mathrm{OH}$ & $\mathrm{NO}_{2}$ & 67 & $197-188$ & $\mathrm{C}_{19} \mathrm{H}_{19} \mathrm{~N}_{3} \mathrm{O}_{4}$ & 353 \\
\hline A11 & $\mathrm{H}$ & $\mathrm{H}$ & $\mathrm{O}----\mathrm{CH}_{2}----\mathrm{O}$ & & 75 & $141-142$ & $\mathrm{C}_{20} \mathrm{H}_{20} \mathrm{~N}_{2} \mathrm{O}_{3}$ & 336 \\
\hline A12 & $\mathrm{H}$ & $\mathrm{OCH}_{3}$ & $\mathrm{H}$ & $\mathrm{OCH}_{3}$ & 73 & $177-179$ & $\mathrm{C}_{21} \mathrm{H}_{24} \mathrm{~N}_{2} \mathrm{O}_{3}$ & 352 \\
\hline A13 & $\mathrm{H}$ & $\mathrm{OCH}_{3}$ & $\mathrm{OCH}_{3}$ & $\mathrm{OCH}_{3}$ & 75 & $215-217$ & $\mathrm{C}_{22} \mathrm{H}_{26} \mathrm{~N}_{2} \mathrm{O}_{4}$ & 382 \\
\hline A14 & $\mathrm{NO}_{2}$ & $\mathrm{H}$ & $\mathrm{H}$ & $\mathrm{H}$ & 85 & 201-203 & $\mathrm{C}_{19} \mathrm{H}_{19} \mathrm{~N}_{3} \mathrm{O}_{3}$ & 337 \\
\hline A15 & $\mathrm{H}$ & $\mathrm{NO}_{2}$ & $\mathrm{H}$ & $\mathrm{H}$ & 86 & $206-208$ & $\mathrm{C}_{19} \mathrm{H}_{19} \mathrm{~N}_{3} \mathrm{O}_{3}$ & 337 \\
\hline
\end{tabular}


TABLE 2. Antituberculosis activity and cytotoxicity of the compounds

\begin{tabular}{|c|c|c|c|c|}
\hline \multirow[t]{2}{*}{ Comp. } & \multicolumn{2}{|c|}{ MABA: $H_{37} R v$ Data } & \multirow{2}{*}{$\begin{array}{c}\text { Cell Titer-Glo: Vero Cell } \\
\mathrm{CC}_{50}(\mu \mathrm{g} / \mathrm{mL})\end{array}$} & \multirow{2}{*}{$\frac{\mathrm{SI}}{\left(\mathrm{CC}_{50} / \mathrm{IC}_{90}\right)}$} \\
\hline & $\mathrm{IC}_{50}(\mu \mathrm{g} / \mathrm{mL})$ & $I C_{90}(\mu \mathrm{g} / \mathrm{mL})$ & & \\
\hline $\mathrm{A} 1$ & 98.743 & $>100$ & - & - \\
\hline $\mathrm{A} 2$ & $>100$ & $>100$ & - & - \\
\hline A3 & $>100$ & $>100$ & - & - \\
\hline A4 & $>100$ & $>100$ & - & - \\
\hline A5 & $>100$ & $>100$ & - & - \\
\hline A6 & $>100$ & $>100$ & - & - \\
\hline A7 & $>100$ & $>100$ & - & - \\
\hline A8 & $>100$ & $>100$ & - & - \\
\hline A9 & $>100$ & $>100$ & - & - \\
\hline A10 & 3.072 & 3.358 & $>40$ & $>11.9$ \\
\hline A11 & $>100$ & $>100$ & - & - \\
\hline $\mathrm{A} 12$ & $>100$ & $>100$ & - & - \\
\hline A13 & $>100$ & $>100$ & - & - \\
\hline A14 & $>100$ & $>100$ & - & - \\
\hline A15 & $>100$ & $>100$ & - & - \\
\hline
\end{tabular}

(1), 307 (11), 291 (1), 188 (12), 186 (20), 171 (12), 159 (23), 151 (64), 145 (100), 129 (26). For $\mathrm{C}_{19} \mathrm{H}_{19} \mathrm{~N}_{3} \mathrm{O}_{3}$ calculated: $67.64 \%$ C, $5.68 \% \mathrm{H}, 12.45 \% \mathrm{~N}$; found: $67.64 \% \mathrm{C}, 5.69 \% \mathrm{H}, 12.47 \% \mathrm{~N}$.

A5: ${ }^{1} \mathrm{H}-\mathrm{NMR}: \delta$ 1.65-1.75 $(4 \mathrm{H}, \mathrm{m}), 2.60-2.75(4 \mathrm{H}, \mathrm{m}), 3.77(2 \mathrm{H}$, s), 6.90-7.05 (3H, m), 7.82-7.90 $(4 \mathrm{H}, \mathrm{m}), 8.17(1 \mathrm{H}, \mathrm{s}), 11.77(1 \mathrm{H}$, s). $\operatorname{EIMS~(m/z):~} 317\left(\mathrm{M}^{+}, 3\right.$ \%), 188 (13), 187 (18), 186 (14), 171 (12), 159 (18), 145 (100), 131 (78). For $\mathrm{C}_{20} \mathrm{H}_{19} \mathrm{~N}_{3} \mathrm{O}$ calculated: $75.69 \%$ C, $6.03 \%$ H, $13.24 \% \mathrm{~N}$; found: $75.71 \% \mathrm{C}, 6.03 \% \mathrm{H}$, $13.27 \% \mathrm{~N}$.

A6: ${ }^{1} \mathrm{H}-\mathrm{NMR}: \delta 1.21(6 \mathrm{H}, \mathrm{d}, J=6.9 \mathrm{~Hz}), 1.65-1.80(4 \mathrm{H}, \mathrm{m}), 2.60-$ $2.75(4 \mathrm{H}, \mathrm{m}), 2.91(1 \mathrm{H}, \mathrm{dt}, J=6.9 \mathrm{~Hz}), 3.73(2 \mathrm{H}, \mathrm{s}), 6.90-7.10(3 \mathrm{H}$, m), $7.31(2 \mathrm{H}, \mathrm{d}, J=8.2 \mathrm{~Hz}), 7.60(2 \mathrm{H}, \mathrm{dd}, J=8.2,3.8 \mathrm{~Hz}), 8.09$ $(1 \mathrm{H}, \mathrm{s}), 11.42(1 \mathrm{H}, \mathrm{s})$. EIMS (m/z): $334\left(\mathrm{M}^{+}, 28 \%\right), 316(1), 290$ (1), 276 (1), 189 (38), 188 (34), 171 (22), 161 (31), 148 (39), 147 (27), 145 (100), 131 (41). For $\mathrm{C}_{22} \mathrm{H}_{26} \mathrm{~N}_{2} \mathrm{O}$ calculated: $79.01 \% \mathrm{C}$, 7.84 \% H, 8.38 \% N; found: $79.05 \%$ C, $7.87 \% \mathrm{H}, 8.40 \% \mathrm{~N}$.

A7: ${ }^{1} \mathrm{H}-\mathrm{NMR}: \delta$ 1.65-1.80 $(4 \mathrm{H}, \mathrm{m}), 2.60-2.75(4 \mathrm{H}, \mathrm{m}), 3.72(2 \mathrm{H}$, s), $5.15(2 \mathrm{H}, \mathrm{s}), 6.90-7.10(5 \mathrm{H}, \mathrm{m}), 7.30-7.50(5 \mathrm{H}, \mathrm{m}), 7.62(2 \mathrm{H}, \mathrm{d}$, $J=8.8 \mathrm{~Hz}), 8.09(1 \mathrm{H}, \mathrm{s}), 11.35(1 \mathrm{H}, \mathrm{s})$. EIMS $(\mathrm{m} / \mathrm{z}): 398\left(\mathrm{M}^{+}, 15\right.$ \%), 307 (1), 253 (16), 225 (9), 212 (3), 188 (9), 171 (5), 145 (45), 91 (100). For $\mathrm{C}_{26} \mathrm{H}_{26} \mathrm{~N}_{2} \mathrm{O}_{2}$ calculated: $78.36 \%$ C, $6.58 \% \mathrm{H}, 7.03 \%$ $\mathrm{N}$; found: $78.38 \% \mathrm{C}, 6.59 \% \mathrm{H}, 7.00 \% \mathrm{~N}$.

A8: ${ }^{1} \mathrm{H}-\mathrm{NMR}: \delta$ 1.60-1.75 $(4 \mathrm{H}, \mathrm{m}), 2.60-2.75(4 \mathrm{H}, \mathrm{m}), 3.75(2 \mathrm{H}$, s), 6.90-7.10 (3H, m), $7.48(1 \mathrm{H}, \mathrm{d}, J=8.5 \mathrm{~Hz}), 7.68(1 \mathrm{H}, \mathrm{s}), 7.90-$ $7.97(1 \mathrm{H}, \mathrm{m}), 8.44(1 \mathrm{H}, \mathrm{s}), 11.74(1 \mathrm{H}, \mathrm{s})$. EIMS $(\mathrm{m} / \mathrm{z}): 360\left(\mathrm{M}^{+}, 5\right.$ \%), 325 (1), 215 (5), 189 (12), 188 (33), 187 (14), 176 (42), 174 (67), 171 (26), 159 (9), 145 (100), 129 (29). For $\mathrm{C}_{19} \mathrm{H}_{18} \mathrm{Cl}_{2} \mathrm{~N}_{2} \mathrm{O}$ calculated: $63.17 \%$ C, $5.02 \% \mathrm{H}, 7.75 \% \mathrm{~N}$; found: $63.19 \% \mathrm{C}, 5.04 \% \mathrm{H}$, $7.71 \% \mathrm{~N}$.

A9: ${ }^{1} \mathrm{H}-\mathrm{NMR}$ : $\delta$ 1.65-1.80 $(4 \mathrm{H}, \mathrm{m}), 2.65-2.80(4 \mathrm{H}, \mathrm{m}), 3.72(2 \mathrm{H}$, s), $3.81(3 \mathrm{H}, \mathrm{s}), 6.82(1 \mathrm{H}, \mathrm{d}, J=8.1 \mathrm{~Hz}), 6.90-7.07(4 \mathrm{H}, \mathrm{m}), 7.26$ $(1 \mathrm{H}, \mathrm{s}), 7.99(1 \mathrm{H}, \mathrm{s}), 9.49(1 \mathrm{H}, \mathrm{s}), 11.30(1 \mathrm{H}, \mathrm{s})$. EIMS $(\mathrm{m} / \mathrm{z}): 338$
$\left(\mathrm{M}^{+}, 49 \%\right), 320$ (12), 294 (1), 193 (36), 188 (25), 171 (17), 166 (47), 165 (40), 145 (100), 129 (28). For $\mathrm{C}_{20} \mathrm{H}_{22} \mathrm{~N}_{2} \mathrm{O}_{3}$ calculated: 70.99 \% C, $6.55 \% \mathrm{H}, 8.28 \% \mathrm{~N}$; found: $70.96 \% \mathrm{C}, 6.59 \% \mathrm{H}, 8.31 \% \mathrm{~N}$.

A10: ${ }^{1} \mathrm{H}-\mathrm{NMR}: \delta 1.65-1.80(4 \mathrm{H}, \mathrm{m}), 2.60-2.75(4 \mathrm{H}, \mathrm{m}), 3.74(2 \mathrm{H}$, s), 6.90-7.05 (3H, m), $7.19(1 \mathrm{H}, \mathrm{dd}, J=8.7,2.1 \mathrm{~Hz}), 7.88(1 \mathrm{H}, \mathrm{dt}$, $J=8.7,2.1 \mathrm{~Hz}), 7.97(1 \mathrm{H}, \mathrm{s}), 8.13-8.20(2 \mathrm{H}, \mathrm{m}), 11.51(1 \mathrm{H}, \mathrm{s})$. EIMS (m/z): $353\left(\mathrm{M}^{+}, 6\right.$ \%), 335 (1), 188 (26), 171 (18), 167 (72), 159 (10), 145 (100), 129 (25). For $\mathrm{C}_{19} \mathrm{H}_{19} \mathrm{~N}_{3} \mathrm{O}_{4}$ calculated: 64.58 \% C, $5.42 \% \mathrm{H}, 11.89 \% \mathrm{~N}$; found: $64.61 \%$ C, $5.45 \% \mathrm{H}, 11.92 \%$ $\mathrm{N}$.

A11: ${ }^{1} \mathrm{H}-\mathrm{NMR}: \delta$ 1.65-1.80 (4H, m), 2.60-2.75 (4H, m), $3.72(2 \mathrm{H}$, s), $6.08(2 \mathrm{H}, \mathrm{s}), 6.90-7.05(4 \mathrm{H}, \mathrm{m}), 7.12(1 \mathrm{H}, \mathrm{dd}, J=8.2,1.6 \mathrm{~Hz})$, $7.25(1 \mathrm{H}, \mathrm{d}, J=1.6 \mathrm{~Hz}), 8.02(1 \mathrm{H}, \mathrm{s}), 11.37(1 \mathrm{H}, \mathrm{s})$. EIMS $(\mathrm{m} / \mathrm{z})$ : $336\left(\mathrm{M}^{+}, 51\right.$ \%), 318 (1), 292 (1), 278 (1), 191 (26), 188 (31), 171 (14), 164 (52), 163 (42), 149 (39), 145 (100), 129 (27). For $\mathrm{C}_{20} \mathrm{H}-$ ${ }_{20} \mathrm{~N}_{2} \mathrm{O}_{3}$ calculated: $71.41 \% \mathrm{C}, 5.99 \% \mathrm{H}, 8.33 \% \mathrm{~N}$; found: 71.44 \% C, $5.95 \% \mathrm{H}, 8.37 \% \mathrm{~N}$.

A12: ${ }^{1} \mathrm{H}-\mathrm{NMR}: \delta 1.65-1.80(4 \mathrm{H}, \mathrm{m}), 2.60-2.75(4 \mathrm{H}, \mathrm{m}), 3.74(2 \mathrm{H}$, s), $3.78(6 \mathrm{H}, \mathrm{s}), 6.55(1 \mathrm{H}, \mathrm{s}), 6.93(2 \mathrm{H}, \mathrm{s}), 6.90-7.05(3 \mathrm{H}, \mathrm{m}), 8.05$ $(1 \mathrm{H}, \mathrm{s}), 11.46(1 \mathrm{H}, \mathrm{s})$. EIMS $(\mathrm{m} / \mathrm{z}): 352\left(\mathrm{M}^{+}, 43 \%\right), 334(1), 308$ (1), 294 (2), 188 (29), 179 (29), 171 (16), 165 (35), 145 (100), 129 (29). For $\mathrm{C}_{21} \mathrm{H}_{24} \mathrm{~N}_{2} \mathrm{O}_{3}$ calculated: $71.57 \%$ C, $6.86 \% \mathrm{H}, 7.95 \% \mathrm{~N}$; found: $71.60 \% \mathrm{C}, 6.89 \% \mathrm{H}, 7.99 \% \mathrm{~N}$.

A13: ${ }^{1} \mathrm{H}-\mathrm{NMR}: \delta 1.65-1.80(4 \mathrm{H}, \mathrm{m}), 2.65-2.77(4 \mathrm{H}, \mathrm{m}), 3.70(3 \mathrm{H}$, s), $3.75(2 \mathrm{H}, \mathrm{s}), 3.82(6 \mathrm{H}, \mathrm{s}), 6.90-7.05(5 \mathrm{H}, \mathrm{m}), 8.06(1 \mathrm{H}, \mathrm{s}), 11.43$ $(1 \mathrm{H}, \mathrm{s})$. EIMS (m/z): $382\left(\mathrm{M}^{+}, 84\right.$ \%), 364 (1), 352 (11), 338 (10), 324 (19), 237 (41), 210 (30), 209 (33), 195 (87), 193 (100), 178 (30), 163 (10), 145 (74), 129 (24). For $\mathrm{C}_{22} \mathrm{H}_{26} \mathrm{~N}_{2} \mathrm{O}_{4}$ calculated: $69.09 \%$ C, $6.85 \% \mathrm{H}, 7.32 \% \mathrm{~N}$; found: $69.12 \%$ C, $6.88 \% \mathrm{H}, 7.35 \% \mathrm{~N}$.

A14: ${ }^{1} \mathrm{H}-\mathrm{NMR}: \delta 1.65-1.80(4 \mathrm{H}, \mathrm{m}), 2.60-2.75(4 \mathrm{H}, \mathrm{m}), 3.74(2 \mathrm{H}$, s), $6.90-7.10(3 \mathrm{H}, \mathrm{m}), 7.60-7.70(1 \mathrm{H}, \mathrm{m}), 7.75-7.83(1 \mathrm{H}, \mathrm{m}), 8.00-$ $8.10(2 \mathrm{H}, \mathrm{m}), 8.51(1 \mathrm{H}, \mathrm{s}), 11.77(1 \mathrm{H}, \mathrm{s})$. EIMS $(\mathrm{m} / \mathrm{z}): 337\left(\mathrm{M}^{+}, 1\right.$ 
\%), 320 (10), 303 (2), 246 (1), 201 (10), 188 (10), 186 (12), 171 (20), 151 (35), 145 (100), 130 (30). For $\mathrm{C}_{19} \mathrm{H}_{19} \mathrm{~N}_{3} \mathrm{O}_{3}$ calculated: 67.64 \% C, $5.68 \% \mathrm{H}, 12.45 \% \mathrm{~N}$; found: $67.66 \% \mathrm{C}, 5.71 \% \mathrm{H}, 12.48 \%$ $\mathrm{N}$.

A15: ${ }^{1} \mathrm{H}-\mathrm{NMR}: \delta 1.65-1.80(4 \mathrm{H}, \mathrm{m}), 2.62-2.75(4 \mathrm{H}, \mathrm{m}), 3.78(2 \mathrm{H}, \mathrm{s})$, 6.90-7.10 (3H, m), 7.68-7.75 (1H, m), 8.08-8.15 (2H, m), 8.20-8.25 $(1 \mathrm{H}, \mathrm{m}), 8.49(1 \mathrm{H}, \mathrm{s}), 11.70(1 \mathrm{H}, \mathrm{s})$. EIMS $(\mathrm{m} / \mathrm{z}): 337\left(\mathrm{M}^{+}, 2 \%\right), 307$ (1), 279 (1), 188 (12), 187 (31), 186 (20), 171 (10), 159 (22), 151 (46), 145 (100), 129 (25). For $\mathrm{C}_{19} \mathrm{H}_{19} \mathrm{~N}_{3} \mathrm{O}_{3}$ calculated: $67.64 \%$ C, $5.68 \%$ $\mathrm{H}, 12.45 \% \mathrm{~N}$; found: $67.69 \% \mathrm{C}, 5.70 \% \mathrm{H}, 12.43 \% \mathrm{~N}$.

\section{Pharmacological evaluation}

Primary Screen (Dose Response)

(Determination of a $90 \%$ Inhibitory Concentration $\left(I C_{90}\right)$ )

The initial screen is conducted against Mycobacterium tuberculosis H37Rv (ATCC 27294) in BACTEC 12B medium using the Microplate Alamar Blue Assay (MABA) (14). Compounds are tested in ten 2-fold dilutions, typically from $100 \mu \mathrm{g} / \mathrm{mL}$ to 0.19 $\mu \mathrm{g} / \mathrm{mL}$. The $\mathrm{IC}_{90}$ is defined as the concentration effecting a reduction in fluorescence of $90 \%$ relative to controls. This value is determined from the dose-response curve using a curve-fitting program. Any $\mathrm{IC}_{90}$ value of $\leq 10 \mu \mathrm{g} / \mathrm{mL}$ is considered "Active" for antitubercular activity. The "Active" compounds are considered for "Secondary Screening".

Secondary Screen

Determination of Mammalian Cell Cytotoxicity $\left(C_{50}\right)$

The VERO cell cytotoxicity assay (15) is done in parallel with the TB Dose Response assay. After 72 hours exposure, viability is assessed using Promega's Cell Titer-Glo Luminescent Cell Viability Assay (16), a homogeneous method of determining the number of viable cells in culture based on quantitation of the
ATP present. Cytotoxicity is determined from the dose-response curve as the CC50 using a curve fitting program. Ultimately, the $\mathrm{CC}_{50}$ is divided by the $\mathrm{IC}_{90}$ to calculate an SI (Selectivity Index) value. SI values of $\geq 10$ are considered for further testing.

\section{RESULTS AND DISCUSSION}

The structures of compounds A1-15 were confirmed by elemental analyses, MS-FAB and ${ }^{1} \mathrm{H}$ - NMR spectral data. All compounds gave satisfactory elemental analysis. The mass spectra (MS (FAB)) of the compounds showed $\mathrm{M}^{+}$peaks, in agreement with their molecular formula.

In the $400 \mathrm{MHz}{ }^{1} \mathrm{H}-\mathrm{NMR}$ spectra of the compounds, the $\mathrm{C}_{6}$ and $\mathrm{C}_{7}$ protons of 5,6,7,8-tetrahydronaphthalene were observed at 1.60-1.80 ppm. The $C_{5}$ and $C_{8}$ protons of 5,6,7,8-tetrahydronaphthalene were observed at $2.60-2.80 \mathrm{ppm}$. The $\mathrm{CH}_{2} \mathrm{CO}$ protons appeared as singlet at 3.70-3.79 ppm. The $\mathrm{N}=\mathrm{CH}$ and $\mathrm{NH}$ protons were observed at 7.90-8.23 ppm and 11.30-11.77 ppm respectively. All the other aliphatic and aromatic protons were observed at expected regions.

The results of antituberculosis and cytotoxicity screening of newly prepared compounds A1-15 are expressed in Table 2. The very important result was observed at antituberculosis activity screening for one of the compounds. The compound A10 showed high antituberculosis activity $\left(\mathrm{IC}_{50}: 3.072 \mu \mathrm{g} / \mathrm{mL}\right.$ and $\left.\mathrm{IC}_{90}: 3.358 \mu \mathrm{g} / \mathrm{mL}\right)$ and low cytotoxicity $\left(\mathrm{CC}_{50}:>40 \mu \mathrm{g} / \mathrm{mL}\right)$. Because of SI value of the compound $\mathbf{A 1 0} \geq 10$, further tests are in progress.

\section{ACKNOWLEDGEMENTS}

Authors are thankful to the Tuberculosis Antimicrobial Acquisition and Coordinating Facility (TAACF) in the USA for the in vitro evaluation of antimycobacterial activity and cytotoxicity.

\section{Bazı yeni hidrazon türevlerinin sentezi ve bunların antitüberküloz etkilerinin değerlendirilmesi}

ÖZET: Heterosiklik hidrazon yapısı antitüberküloz aktiviteleri nedeniyle medisinal kimyacıların dikkatini çeken, cazip biyolojik olarak aktif önemli bir ilaç sınıfıdır. Bu amaçla, yeni hidrazon türevleri sentezlendi ve antitüberküloz etkinlikleri değerlendirilmiştir. (5,6,7,8-Tetrahidronaftalen-1-il) asetik asit hidraziti ile çeşitli benzaldehitlerin reaksiyonu, 5,6,7,8-tetrahidronaftalen asetik asit benziliden hidrazit türevlerini verdi. Bileşiklerin kimyasal yapıları 1H-NMR, EI-MS spectral verileri ve elemental analiz metodları ile aydınlatıldı. BACTEC 460 radyometrik sistem ve BACTEC 12B ortamından yararlanılarak Mycobacterium tuberculosis H37Rv (ATCC 27294)'e karşı bileşiklerin antitüberküloz aktiviteleri değerlendirilmiştir. Bileşik A10 yüksek antitüberküloz etkinlik (IC50: $3.072 \mu \mathrm{g} / \mathrm{mL}$ ve IC90: $3.358 \mu \mathrm{g} / \mathrm{mL}$ ) ve düşük sitotoksisite (CC50: $>40 \mu \mathrm{g} / \mathrm{mL}$ ) gösterdi.

ANAHTAR KELIMELER: Hidrazon, Antitüberküloz etki, Mycobacterium tuberculosis H37Rv

\section{REFERENCES}

1. Meng Q, Luo H, Chen $Y$, Wang $T$, Yao Q. Synthesis of novel [1,2]-diamines with antituberculosis activity. Bioorg Med Chem Lett, 19: 5372-5375, 2006.

2. Kaymakçıŏ̆lu BK, Oruç E, Unsalan S, Kandemirli F, Shvets N, Rollas S, Dimoglo A. Synthesis and characterization of novel hydrazide-hydrazones and the study of their structure-antituberculosis activity. Eur J Med Chem, 44: 1253-1261, 2006.
3. Kaymakçıŏlu BK, Rollas S. Synthesis, characterization and evaluation of antituberculosis activity of some hydrazones. Farmaco, 57: 595-599, 2002.

4. Swamy BN, Suma TK, Rao GV and Reddy GC. Synthesis of isonicotinoylhydrazones from anacardic acid and their in vitro activity against Mycobacterium smegmatis. Eur J Med Chem, 42: 420-424, 2007.

5. Küçükgüzel SG, Rollas S. Synthesis, characterization of novel coupling products and 4-arylhydrazono-2-pyra- 
zoline-5-ones as potential antimycobacterial agents. Farmaco, 57: 583-587, 2002.

6. Sriram D, Yogeeswari P, and Madhu K. Synthesis and in vitro and in vivo antimycobacterial activity of isonicotinoyl hydrazones. Bioorg Med Chem Lett, 15: 4502-4505, 2005.

7. Sriram D, Yogeeswari P, and Devakaram RV. Synthesis, in vitro and in vivo antimycobacterial activities of diclofenac acid hydrazones and amides. Bioorg Med Chem, 14: 3113-3118, 2006.

8. Patole J, Sandbhor U, Padhye S, Deobagkar DN, Anson CE, Powell A, Structural chemistry and in vitro antitubercular activity of acetylpyridine benzoyl hydrazone and its copper complex against Mycobacterium smegmatis. Bioorg Med Chem Lett, 13: 51-55, 2003.

9. Maccari R, Ottana R, and Vigorita MG, In vitro advanced antimycobacterial screening of isoniazid-related hydrazones, hydrazides and cyanoboranes: Part 14. Bioorg Med Chem Lett, 15: 2509-2513, 2005.

10. Turan-Zitouni G, Özdemir A, Kaplancıklı ZA, Benkli K, Chevallet P and Akalın G, Synthesis and antituberculosis activity of new thiazolylhydrazone derivatives. Eur J Med Chem, 43: 981-985, 2008.

11. Çukurovalı A, Yılmaz I, Gür S, Kazaz C, Synthesis, antibacterial and antifungal activity of some new thiazolylhydrazone derivatives containing 3-substituted cyclobutane ring. Eur J Med Chem, 41: 201-207, 2006.
12. Hamzacebi MC, Rollas $S$, Küçükgüzel SG, Kaymakçığlu BK, Synthesis and structure elucidation of hydrazones derived from N-(2,4-dimethylphenyl)-3-oxobutanamide. Arkivoc, 12: 188-194, 2008.

13. Karalı N, Kocabalkanlı A, Gürsoy A, Ateş Ö, Synthesis and antitubercular activity of 4-(3-coumarinyl)-3-cyclohexyl-4-thiazolin-2-one benzylidenehydrazones. Farmaco, 57: 589-593, 2002.

14. Yale HL, Losee $K$, Martins J, Holsing M, Perry FM, Bernstein J, Chemotherapy of experimental tuberculosis. VIII. The synthesis of acid hydrazides, their derivatives and related compounds. J Am Chem Soc, 75: 1933-1942, 1953.

15. Collins LA, Franzblau SG, Microplate alamar blue assay versus BACTEC 460 system for high-throughput screening of compounds against Mycobacterium tuberculosis and Mycobacterium avium. Antimicrob Agents Chemother, 41: 1004-1009, 1997.

16. Lara-Díaz VJ, Gaytán-Ramos AA, Dávalos-Balderas AJ, Santos-Guzmán J, Mata-Cárdenas BD, Vargas-Villarreal J, Barbosa-Quintana A, Sanson M, López-Reyes AG, Moreno-Cuevas JE. Microbiological and toxicological effects of perla black bean (Phaseolus vulgaris L.) extracts:in vitro and in vivo studies. Basic Clin Pharmacol Toxicol, 104: 81-86, 2009.

17. CellTiter-Glo® Luminescent Cell Viability Assay, Promega Corporation's, http://www.promega.com/ tbs/tb288/tb288.html June 2009. 